\title{
Detection of Pulsed Radar in a Time Division Duplexed System
}

\author{
Brad W. Zarikoff \\ Hamilton Institute \\ National University of Ireland Maynooth \\ Email: Brad.Zarikoff@nuim.ie
}

\author{
David Weldon \\ Wireless R\&D, Vecima Networks, Inc. \\ Victoria, BC, Canada V8Z 3B8 \\ Email: David.Weldon@Vecima.com
}

\begin{abstract}
Dynamic Frequency Selection (DFS) is the technology chosen by ETSI, FCC, and Industry Canada to provide unlicensed access for broadband radios in licensed bands. It is implemented by the basestation or basestation/subscriber combination. In a time-division duplexed (TDD) system with DFS implemented solely at the basestation, a periodic 'quiet time' is enforced. This work determines the likelihood that a radar pulse train can be detected in a TDD system with radar detection restricted to the basestation. The results are tailored for TDD systems such as WiMAX, and can be used by designers to determine under what system constraints DFS can be implemented in their systems.
\end{abstract}

\section{INTRODUCTION}

Recently, the Federal Communications Commission (FCC) has opened the 5250 to $5350 \mathrm{MHz}$ and 5470 to $5725 \mathrm{MHz}$ bands to unlicensed broadband wireless use; in Europe, the 5470 to $5725 \mathrm{MHz}$ European Telecommunications Standards Institute (ETSI) band is similarly used. As these bands are traditionally used for radar purposes (primarily for weather and military uses), the FCC and ETSI has imposed certain restrictions for unlicensed broadband wireless use [1], [2], and refer to these restrictions as dynamic frequency selection (DFS). Essentially, these restrictions require the unlicensed communications equipment to spend time sensing the active channel for radar presence, before a session is established and during the session; this period is referred to as the 'Channel Availability Check Time' and is 60 seconds in duration (FCC and ETSI). During the communications session, the BS must continuously look for radar pulses. In the case that a radar burst is detected, the basestation (BS) must organize the communications session to be relocated to another channel. The 'Channel Closing Transmission Time' is the period allocated during which all transmissions must cease (200 milliseconds for FCC and 1 second for ETSI). The 'Channel Move Time' is the period allocated during which the communications session must be re-established in another channel, and is 10 seconds in duration (FCC and ETSI). After the BS has vacated the channel, the channel must be left vacant for a specific time period; this period is referred to as the 'Non-Occupancy Period', and is 30 minutes in duration.

This work was done at Vecima Networks and is supported by Science Foundation Ireland under Grant No. 08/SRC/I1403. Submitted to the 2011 IEEE $73^{\text {rd }}$ Vehicular Technology Conference (VTC-2011 Spring).
In a time-division duplexed (TDD) system, radar detection is typically done with sampled data from the RF receive chain. However, one of the main caveats in formulating a DFS system for a TDM/TDMA scheme such as WiMAX is that the receive chain must be muted during transmission. This cannot be avoided by using a secondary receive chain, since the transmitter will still drown out any signal into the secondary receiver. Hence, the DFS system must account for this inherent 'quiet time'. At the moment, this can only be done by judicious selection of the ratio between the uplink and downlink (receive and transmit at the BS). Since the receive mute duration is deterministic, but the radar pulse train (referred to as a radar burst) arrival time is random, it is possible to determine the statistical overlap of a specific radar burst and the receive frame of the WiMAX system. This provides the focus of this paper: determining the probability of a radar pulse landing within the receive portion of a WiMAX frame. We chose the sample radar types given in the FCC test procedure [3] to provide examples.

Surprisingly, the current literature is silent on analysis of radar detection probabilities. Calculation of radar detection probabilities used in the FCC and ETSI standards would have required simulations at the minimum. Literature on DFS for radar appears restricted to detection algorithms [4] and policy review [5].

Since the test radar waveforms as specified by the FCC and ETSI are sent in pulse trains, they inherently provide a means to avoid false positives due to random interference. An intelligent algorithm that takes the pulse widths and spacing after initial pulse identification is necessary to determine whether a radar pulse is present or not. For this work, we assume that a number of consecutive radar pulses must be identified in order for the algorithm to be successful, and thus we must find the probability of a number of consecutive pulses landing within the receive portion of the WiMAX frame.

\section{BACKGROUND}

To make better use of the limited RF spectrum, government spectrum sharing in the form of dynamic frequency selection (DFS) started in 2004 [5]. The basic premise of DFS is to allow private users access to the under-utilized radar bands in the $5 \mathrm{GHz}$ range. Because of the demand that government users place on the probability of radar detection, the private 


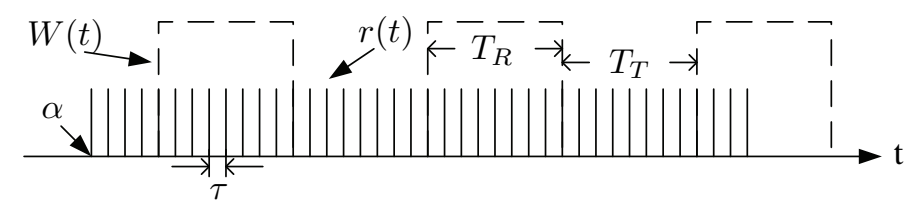

Fig. 1. Signal Model

users access to these bands are subject to them implementing quite strict cognitive-radio-based sharing techniques.

Since the DFS standards were introduced, studies have shown the effectiveness of co-existence of radar and wideband communication signals [6]. In [6], DFS was tested using an IEEE 802.11a RLAN system operating in the vicinity of a Doppler weather radar system. The conclusion was that the RLAN introduced additive, uncorrelated noise, from the radars perspective. The authors note that the DFS would detect the radar sooner than the RLAN would corrupt the radar.

As mentioned in the introduction, a TDD system that implements DFS will experience some muted periods during which the receiver is turned off. This work focuses on determining the probability that a portion of a radar burst will arrive at a BS during the period when it is in receive mode, and thus the probability that specific radar types can be detected in TDD mode.

\section{A. System Model}

Our model consists of two signals: that of the periodic transmit/receive modes of the BS and that of the arriving radar pulse. Power levels are not considered. Fig. 1 demonstrates a sample composite waveform, where a single sample test radar waveform consisting of forty pulses overlaps three consecutive receive frames. Each TDD frame is of duration $T$. On any given frame, the first $T_{T}$ seconds is for transmit, while the remaining $T_{R}=T-T_{T}$ is for receive, during which radar detection is possible. We use a continuous-time sampling function, $W(t)$, to denote the receive window of the TDD radio; during the receive portion of a frame, $W(t)=1$, otherwise, $W(t)=0$. The radar burst arrival time is $\alpha \in$ $[0, T]$, with the radar waveform itself denoted $r(t)$. The radar bursts consist of $N$ pulses, each separated by $\tau$ seconds, where $\tau$ is the PRI. The total duration of the radar pulse train is then $T_{P}=(N-1) \tau$. The variable $P$ is the number of pulses that land in $W(t)$.

\section{B. Assumptions}

A number of assumptions and definitions are used to simplify the following analysis. For the radar burst, the pulses considered are taken from the FCC and ETSI standards, and can vary in duration from $1 \mu s$ to $20 \mu s$. To simplify the math, this analysis assumes that they are unit impulses. Justification for this can be made by examining the ratio of the pulse repetition interval (PRI) to the pulse width, which varies from about 10 to 1428; this relates to a duty cycle of $10 \%$ to $0.07 \%$. We also assume that for a single radar burst arriving in the current interval $[0, T]$, the arrival time $\alpha$ is uniformly

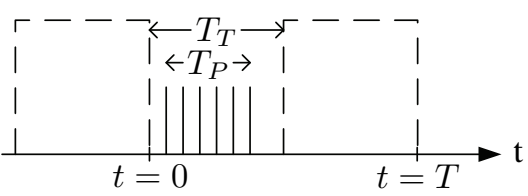

Fig. 2. Scenario for Case 1: $T_{P}<T_{T}$

distributed, and that the PRI $\tau$ lies in $\left[\tau_{\min }, \tau_{\max }\right]$ and is also uniformly distributed. These assumptions are considered valid given that we only consider a single radar burst. In real systems, there may be subsequent bursts, but this case is not considered in this work.

\section{Pulse Detection}

Our goal is to find the probability that $n$ radar pulses of a given radar type land during the receive portion of a TDD frame (while $W(t)$ has a value of 1). The system designer can use these techniques to specific limits on the receive-totransmit ratio of the radio system and determine the maximum number of pulses the radar detection algorithm should search for [4]. First, we will attempt some analysis on two special cases: Case 1, where the radar pulse train is less than $T_{T}$ and therefore will only effect a single frame, and Case 2, if the radar pulse train is greater than $T+T_{R}$, and therefore will effect at least two frames. In both cases, we will consider only a single frame, so the domain of $W(t)$ is restricted to $t \in[0, T]$. Then, to accommodate for a greater variety of radar types, a more detailed simulation model will be devised and used for comparison.

As a matter of notational convenience, the probabilities $\operatorname{Pr}(P=N)$ are conditioned on the total duration of the incoming radar burst $T_{P}$. In this work, the radar types are not considered as random variables, and thus the conditional statements do not portray any stochastic dependence.

\section{A. Case 1: $T_{P}<T_{T}$}

Case 1 itself can be divided up into two scenarios: if the entire radar pulse lands within $W(t)$, and if less than $N$ pulses land within $W(t)$. As mentioned in Section II-B, we assume that $\alpha$ is uniformly distributed and that the pulses are impulse functions. This means that, given that $\alpha$ is distributed from $[0, T]$, the probability of $\alpha$ being within $W(t)$ (or $\left(T_{T} \leq \alpha \leq\right.$ $T)$ ) is $\left(T-T_{T}\right) / T$ or $T_{R} / T$.

It is possible that not all radar pulses will fit within $W(t)$. The maximum number of pulses that can land in $W(t)$ can be found as the ratio between $T_{R}$ and $\tau$ to be

$$
X_{P}=\left\lceil T_{R} / \tau\right\rceil
$$

If $X_{P} \geq N, \operatorname{Pr}\left(P=N \mid T_{P}<T_{T}\right)$ is non-zero, where $N$ is the number of pulses in $r(t)$ and $P$ is the number of pulses landing in $W(t)$. Using the above result for the probability of $\alpha$ landing in $W(t)$, we can find the probability that all $N$ radar pulses land within $W(t)$ when $T_{P}<T_{R}$ to be

$$
\begin{aligned}
\operatorname{Pr}\left(P=N \mid T_{P}<T_{T}\right) & =\left(T_{R}-T_{P}\right) / T \\
& =R_{R}-T_{P} / T,
\end{aligned}
$$




$$
\begin{aligned}
\operatorname{Pr}\left(P \leq X_{P}-1 \mid T_{P}>T+T_{R}\right) & =1 \\
\operatorname{Pr}\left(P=X_{P} \mid T_{P}>T+T_{R}\right) & =\frac{T_{R} \log \left(\tau_{\max }-\tau_{\min }\right)}{\tau_{\max }-\tau_{\min }}-X_{P}+1 \\
\operatorname{Pr}\left(P>X_{P} \mid T_{P}>T+T_{R}\right) & =0 .
\end{aligned}
$$

and 0 otherwise. The receive to full frame ratio is defined as $R_{R}=T_{R} / T$. We can see that if $T_{P} \ll T$, then this probability reduces to $R_{R}$, while if $T_{P}>T_{R}$, then the probability goes to zero.

If $X_{P}<N$, we must start with finding the probability that $P=X_{P}$. This probability is dependent on the number of pulses that will not fall in $W(t)$ when $P=X_{P}$, which is $N-X_{P}$. To calculate $\operatorname{Pr}\left(P=X_{P} \mid T_{P}<T_{T}\right)$, define an excess time $\epsilon$ which is the slack in the burst arrival time during which $P=X_{P}$ as

$$
\epsilon=T_{R}-\left(X_{P}-1\right) \tau .
$$

Normalizing $\epsilon$ by $T$ gives the probability of $P=X_{P}$ for a single set of pulses (i.e. the first $X_{P}$ pulses). Then, considering that there are $N-X_{P}$ pulses that do not fall in $W(t)$, the probability that $P=X_{P}$ for all combinations is

$$
\operatorname{Pr}\left(P=X_{P} \mid T_{P}<T_{T}\right)=\left(N-X_{P}+1\right) \epsilon / T
$$

The probability that $P<X_{P}$ must be found both for when the last $n$ pulses lie within the start of $W(t)$ and when the first $n$ pulses lie within the end of $W(t)$. Also, since the pulses are evenly distributed within a single pulse train, the probability of one pulse landing in $W(t)$ is the same as two pulses landing in $W(t)$, and so on. Then we can work out $P<X_{P}$ to be

$$
\begin{aligned}
& \operatorname{Pr}\left(P=p \mid p<X_{P}, T_{P}<T_{T}\right)= \\
& \quad \operatorname{Pr}\left(T_{T}-\tau \leq \alpha \leq T_{T}\right)+ \\
& \quad \operatorname{Pr}\left(T-T_{P}+\tau \leq \alpha \leq T-T_{P}+2 \tau\right) \\
& \quad=2 \tau / T .
\end{aligned}
$$

By summing over the probabilities, we can break the probability of $n$ or more pulses landing in $W(t)$ into two expressions: conditioned on $X_{P} \geq N$ and $X_{P}<N$, so we get

$$
\begin{gathered}
\operatorname{Pr}\left(P \geq n \mid X_{P} \geq N, T_{P}<T_{T}\right)= \\
\operatorname{Pr}\left(P=N \mid T_{P}<T_{T}\right)+ \\
\sum_{i=n}^{N-1} \operatorname{Pr}\left(P=i \mid T_{P}<T_{T}\right), \\
\operatorname{Pr}\left(P \geq n \mid X_{P}<N, T_{P}<T_{T}\right)= \\
\operatorname{Pr}\left(P=X_{P} \mid T_{P}<T_{T}\right)+ \\
\sum_{i=n}^{N-1} \operatorname{Pr}\left(P=i \mid T_{P}<T_{T}\right) .
\end{gathered}
$$

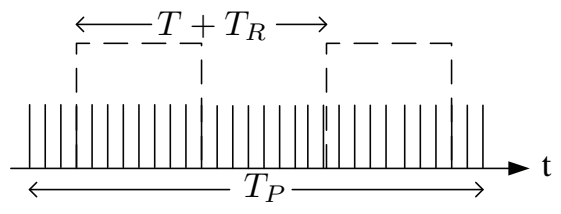

Fig. 3. Scenario for Case 2: $T_{P}>T+T_{R}$

To include the random nature of the PRI $\tau$ and the number of pulses $N$ [3], we take the expectation of (7) and (8) with respect to $\tau$ and $N$. Doing so, we get

$$
\begin{aligned}
& \operatorname{Pr}\left(P \geq n \mid X_{P} \geq N, T_{P}<T_{T}\right)= \\
& \quad R_{R}-(\bar{N}-1) \bar{\tau} / T+2(\bar{N}-1) \bar{\tau} / T, \\
& \operatorname{Pr}\left(P \geq n \mid X_{P}<N, T_{P}<T_{T}\right)= \\
& \quad\left(\bar{N}-X_{P}+1\right) \bar{\epsilon} / T+2(\bar{N}-1) \bar{\tau} / T,
\end{aligned}
$$

where $\bar{\tau}=\mathrm{E}[\tau]$ and $\bar{N}=\mathrm{E}[N]$.

B. Case 2: $T_{P}>T+T_{R}$

In this Case, one receive frame in $W(t)$ is guaranteed to completely overlap with the radar burst $\left(X_{P}<N\right)$. Also, as long as $\tau<T_{R}$, at least one pulse of the pulse train is guaranteed to land. The probability of $P=X_{P}$ can be calculated by using the fact that the pulses are equi-spaced and initially land with uniform probability, so

$$
\begin{aligned}
\operatorname{Pr}\left(P=X_{P} \mid T_{P}>T+T_{R}\right) & =T_{R} / \tau-\left\lfloor T_{R} / \tau\right\rfloor \\
& =T_{R} / \tau-\left(X_{P}-1\right),
\end{aligned}
$$

which is the ratio remaining after removing the $X_{P}-1$ pulses that are guaranteed to land in $W(t)$. The reason that Case 1 and Case 2 are not equal when $P=X_{P}$ is because in Case 1 , the domain that $\alpha$ can occupy in order for $P=X_{P}$ is $\alpha \in\left[T-T_{P}, T_{T}\right]$, while the domain for Case 2 is $\alpha \in[0, T]$. The probability that $P>X_{P}$ pulses land in $W(t)$ is zero because of the equal spaced pulses, and the probability that $P=X_{P}-1$ is $1-\operatorname{Pr}\left(P=X_{P} \mid T_{P}>T+T_{R}\right)$, on account that either $X_{P}$ or $X_{P}-1$ pulses are guaranteed to land in $W(t)$ in a frame.

After taking the expectation with respect to $\tau$, the conditional probabilities are provided for $T_{P}>T+T_{R}$ in (2).

\section{Simulation Model}

Since the radar types defined in [2] and [3] have random components and will usually not fit into either of the Case 1 or Case 2 categories, a more detailed model is created. Using the random radar burst arrival time $\alpha$ and the random PRI $\tau$ 


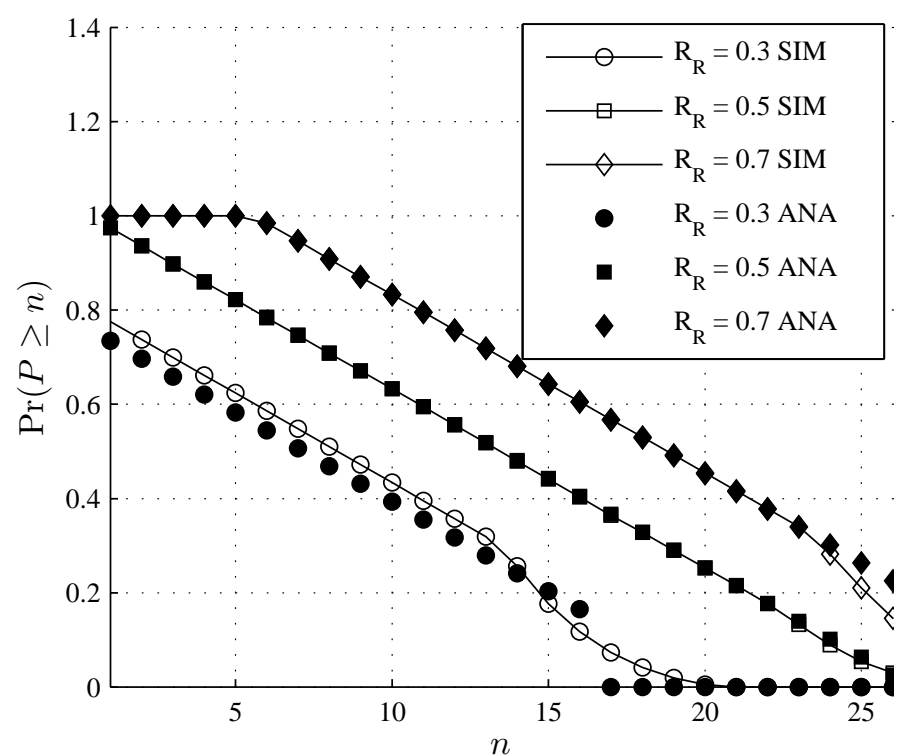

Fig. 4. Probability of $\geq n$ FCC type 2 pulses landing in $W(t)$

as shown in Fig. 1, we can represent the full radar pulse train $r(t)$ as the sum of Dirac delta functions

$$
r(t)=\sum_{n=1}^{N} \delta(t-(n-1) \tau-\alpha)
$$

To determine the number of pulses that land in $W(t)$, the integral of $W(t) r(t)$ can be taken as

$$
P(\alpha, \tau)=\int_{T_{T}}^{T} W(t) r(t) d t
$$

Note that if $T_{P}>T_{T}$, then some pulses may land in subsequent receive frames. This case is not considered in the analysis of the Case 1 and Case 2, and thus we can expect the simulated results using (13) to be more accurate (i.e. more pulses may be captured in subsequent frames).

\section{RESUlts AND Simulations}

We consider the test radar pulses described in [3], specifically Radar Types 1, 2, 3, 4 and 6. FCC Radar Type 5 is not considered as it is a chirp pulse, and therefore significantly different in structure than the others. For Radar Type 6, we consider only a single burst of nine pulses. The parameters of these test pulses are given in Table I, including the required detection probability rate (Det. Prob.) in the FCC specification [3]. Note that for Radar Types 1 through 4, the aggregate detection probability is shown; the minimum probability is $60 \%$. For calculation of (13), the total length of the burst is considered, and any pulses that land in subsequent frames are included in the results. This result is compared to the analytical results of Case 1 (for Radar Types 2, 3, 4, and 6) and of Case 2 (for Radar Type 1). The simulation results are shown after Monte Carlo simulation over $N$ using $10^{4}$ samples, with $N$, $\alpha$ and $\tau$ being uniformly distributed.

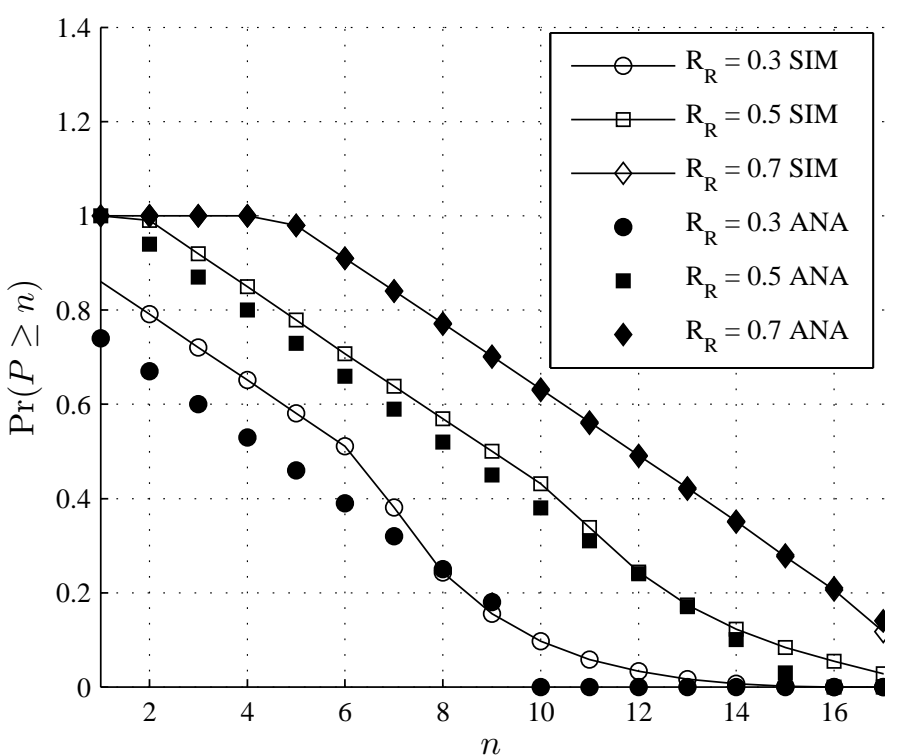

Fig. 5. Probability of $\geq n$ FCC type 3 pulses landing in $W(t)$

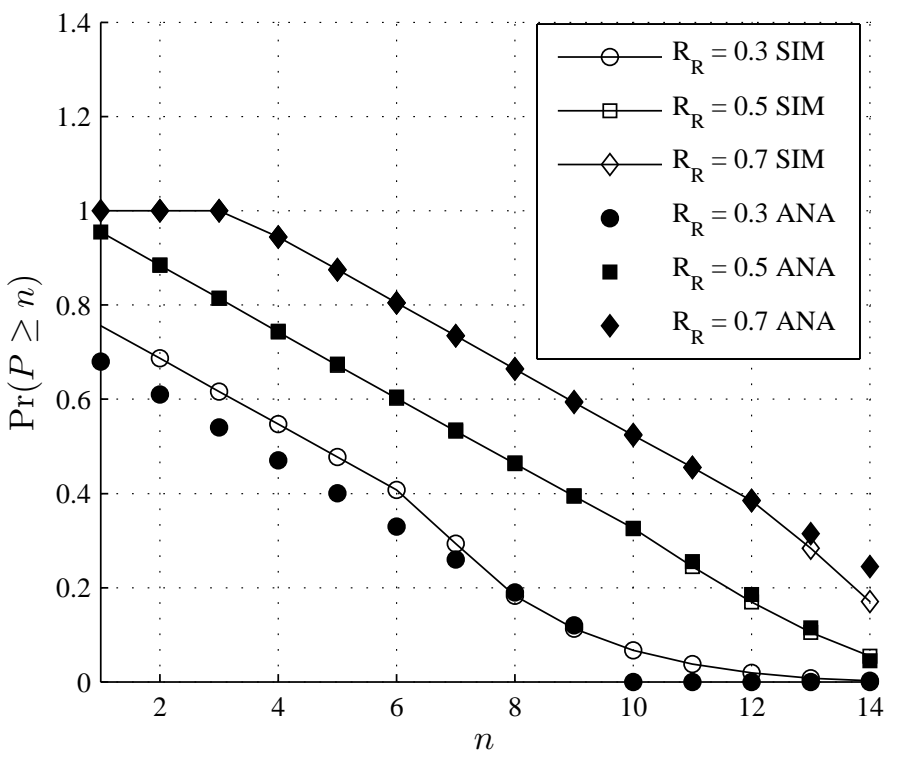

Fig. 6. Probability of $\geq n$ FCC type 4 pulses landing in $W(t)$

Monte Carlo simulation using (13) is used to generate values for $\operatorname{Pr}(P \geq n)$, using $10^{4}$ samples. Fig. 4, 5, 6, and 7 show $\operatorname{Pr}(P \geq n)$ (referenced with SIM) and $\operatorname{Pr}\left(P \geq n \mid T_{P}<T_{T}\right)$ (referenced with ANA) for each of the test pulses. Curves for $R_{R}=0.3,0.5$, and 0.7 are shown, with $T=10 \mathrm{~ms}$. Obviously, as $R_{R}$ increases, the probability of seeing a pulse increases, as the muted portion of the frame is shortened. However, by reducing $R_{R}$, the proportion of uplink to downlink traffic is reduced, with ramifications for load balancing.

It may be argued that a minimum of three pulses are required to identify a radar burst; any less may be noise. A thorough analysis involving SNR and threshold values is required to verify this rule of thumb, but laboratory experi- 


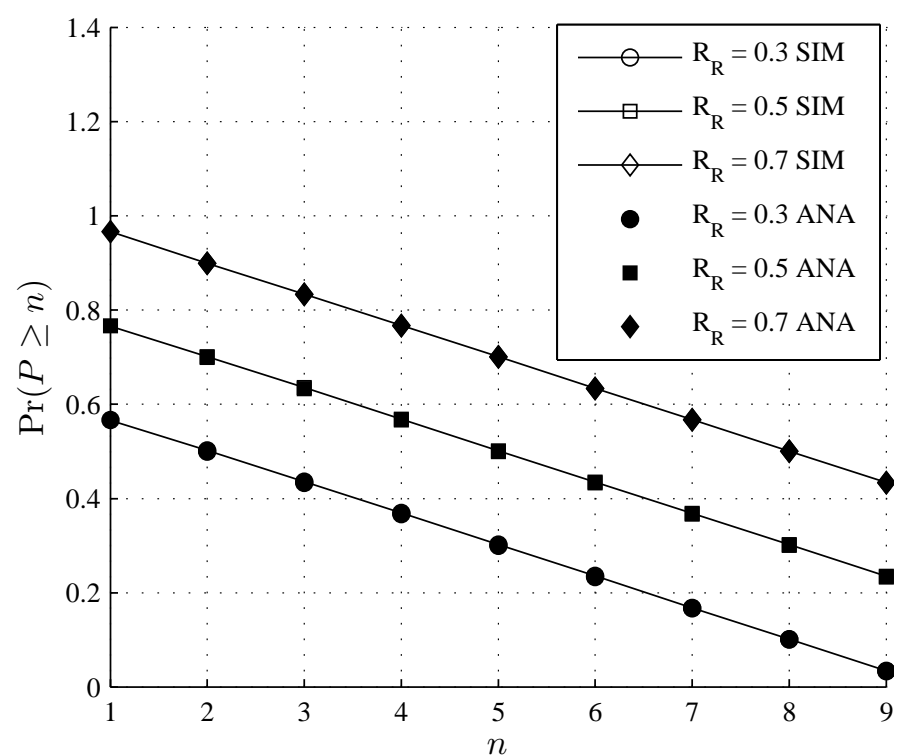

Fig. 7. Probability of $\geq n$ FCC type 6 pulses landing in $W(t)$

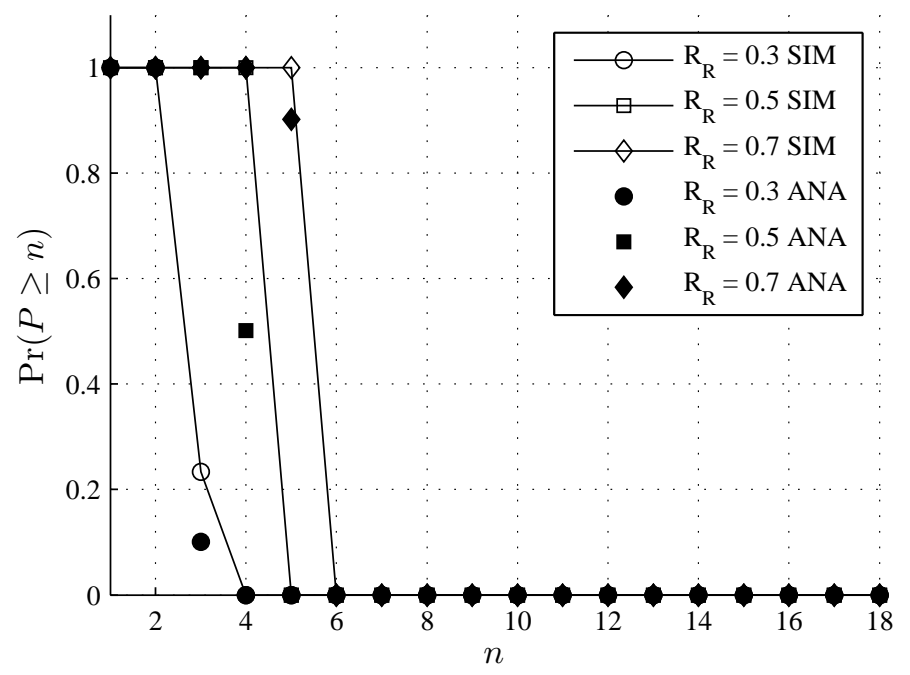

Fig. 8. Probability of $\geq n$ FCC type 1 pulses landing in $W(t)$

ments provide evidence of this, and so we will use this value in the following example. For $R_{R}=0.5, \operatorname{Pr}(P=3)$ is approximately $90 \%$ for Radar Type 2 . Similarly, $\operatorname{Pr}(P=3)$ is approximately $90 \%$ for Radar Type 3 and $80 \%$ for Radar type 4. Due to the short burst duration of Radar Type 6, there is only a $65 \%$ probability that three pulses will land in $W(t)$. Note that for Radar Type 3 and 4 , if $R_{R}=0.7, \operatorname{Pr}(P=3)$ is $100 \%$. This results from a combination of a large $T_{P}$ and $T_{R}$.

The analytical results follow the simulation results closely. As expected, the general trend is for the analytical results to show a lower probability of pulse detection, given that subsequent frames are not considered. However, since our analysis includes an average over $N$ and $\tau$, there is some variation, particularly when the probability of detecting greater than $n$ pulses is low.

For Case 2, Radar Type 1 is evaluated. Due to the large PRI
TABLE I

FCC Test Pulse Parameters

\begin{tabular}{c|c|c|c|c}
\hline Test Pulse & Pulse Width $(\mu s)$ & $\tau(\mu s)$ & N & Det. Prob. \\
\hline \hline 1 & 1 & 1428 & 18 & $80 \%$ \\
\hline 2 & $1-5$ & $150-230$ & $23-29$ & $80 \%$ \\
\hline 3 & $6-10$ & $200-500$ & $16-18$ & $80 \%$ \\
\hline 4 & $11-20$ & $200-500$ & $12-16$ & $80 \%$ \\
\hline 6 & 1 & 333 & 9 & $70 \%$ \\
\hline \multicolumn{4}{|r}{}
\end{tabular}

of Radar Type 1, there is no way that more than four pulses can be detected within a single $5 \mathrm{~ms}$ receive frame. Subsequent receive frames can be used to help in radar pulse identification, or, a lower number of pulses can be used. However, the odds are good that a small number of pulses will be seen: there is a $100 \%$ chance that three pulses will land in a receive frame for $T_{R}=5 \mathrm{~ms}$.

\section{CONCLUSIONS}

Due to the general nature of time division duplexed (TDD) systems, implementation of dynamic frequency selection (DFS) techniques will need to deal with periodic down time when the local transmitter is active. However, radar pulses may still be arriving during these down times. This paper provides an analysis on the probability of whether a radar pulse train can be detected in the case of TDD systems. Using the example radar pulse trains specified by the $\mathrm{FCC}$ for the $5 \mathrm{GHz}$ U-NII bands, simplified expressions for pulse observations are provided. The analysis and simulations provide the system designer with tools to decide which specific transmit to receive ratios are compatible with the DFS limits specified by the FCC and ETSI. Future work will include finding optimal values for transmit and receive ratios. Since the transmit time is restricted by DFS in TDD systems, the concept of further interleaving the transmit and receive windows will also be considered.

\section{REFERENCES}

[1] FCC, Title 47 CFR, Part 15 Subpart E, Federal Communications Commission, 2008.

[2] ETSI, ETSI EN 301 893, European Telecommunications Standards Institute, 2008.

[3] FCC, Appendix: Compliance measurement procedures for UnlicensedNational Information Infrastructure devices operating in the 5250-5350 $\mathrm{MHz}$ and 5470-5725 MHz bands incorporating Dynamic Frequency Selection, Federal Communications Commission, 2006.

[4] M. Wen and L. Hanwen, "Radar detection for 802.11a systems in $5 \mathrm{GHz}$ band," in IEEE Int. Conf. on Wireless Commun., Networking and Mobile Computing, 2005, pp. 23-26.

[5] M. J. Marcus, "Sharing government spectrum with private users: opportunities and challenges," IEEE Wireless Commun., vol. 16, no. 3, pp. 4-5, 2009.

[6] A. L. Brandao, J. Sydor, and W. Brett, "5 GHz RLAN interference on active meteorological radars," in IEEE 61st Veh. Tech. Conf., 2005, pp. $1328-1332$. 